\title{
Reflexiones en el tránsito hacia la pospandemia desde experiencias estudiantiles y docentes en una universidad pública argentina
}

\section{Reflections on the transition to the post-pandemic from student and teacher experiences in an Argentine public university}

\section{María Paula Pierella}

https://orcid.org/0000-0002-6679-9869

paupierella@gmail.com

Instituto Rosario de Investigaciones en Ciencias de la Educación (IRICE-CONICET/UNR) |

Universidad Nacional de Rosario | Argentina

\author{
Ana Borgobello \\ https://orcid.org/0000-0002-2340-8127 \\ borgobelloana@gmail.com \\ Instituto Rosario de Investigaciones en Cien- \\ cias de la Educación (IRICE-CONICET/UNR) | \\ Universidad Nacional de Rosario | Argentina
}

\section{RESUMEN}

En este artículo compartimos algunas reflexiones surgidas en la confluencia de investigaciones sobre el ingreso a la universidad en tiempos de pandemia y procesos de formación docente universitaria desarrollados en ese marco. Dentro de las vastas dimensiones abiertas en este contexto de transformaciones, nos centramos en las condiciones a partir de las cuales se acercaron estudiantes ingresantes y docentes a la virtualización forzosa, advirtiendo sobre las problemáticas en cuanto a las desigualdades en torno al acceso y la formación pedagógica y tecnológica. Nos detenemos luego en las experiencias educativas virtualizadas, enfatizando en la dimensión del conocimiento. Seguidamente, introducimos la problemática del reconocimiento, que consideramos nodal para pensar los vínculos entre estudiantes y docentes, especialmente en los primeros años. A modo de cierre, nos preguntamos acerca del tránsito desde la crisis al después en términos de las posibilidades de apropiación de espacios en tiempos de pospandemia.

\section{ABSTRACT}

This paper emerged from the confluence of research on university admission and teacher training processes during pandemic times. Within the vast dimensions opened in this context of transformations, we focused on the fresh students' conditions and teachers' approaches to forced virtualization, warning about the problems regarding inequalities around access and pedagogical and technological training. We then emphasized on educational experiences about knowledge. Next, we introduced the problem of recognition considering the links between students and teachers, especially in the first year at university level. Finally, we asked ourselves about transition and later possibilities of space appropriation in times post-pandemic.

PALABRAS CLAVE

Virtualización Forzosa Ingreso a la Universidad, Permanencia, Formación Docente.

\section{KEY WORDS}

Virtualization, University Admission, Permanence, Teacher Training 


\section{INTRODUCCIÓN}

Desde comienzos de 2020 el mundo se enfrentó a una situación sin precedentes a partir de la pandemia COVID19. A partir de ello y del consecuente aislamiento social, preventivo y obligatorio (ASPO), las actividades diarias fueron profundamente modificadas, siendo la educación, una de las instituciones más afectadas.

Luego del primer impacto en el que todas las coordenadas se alteraron, comenzaron las preguntas. En nuestro caso, como docentes universitarias e investigadoras cuyos temas de indagación se relacionan con el ingreso a la universidad y la virtualización de la vida académica, estos interrogantes se orientaron específicamente a desentrañar cómo estarían viviendo la situación de pandemia quienes recién se asomaban a dicha institución. También nos interesaba indagar cómo se las estaría "arreglando" la docencia universitaria cuando, de un modo tan intempestivo, se vieron (nos vimos) en la obligación de reinventar formas de enseñar, de diseñar, de presentar los contenidos con la convicción de que, de algún modo, había que estar ahí, haciéndole frente al desconcierto.

En este marco, el interés que orienta el presente texto es compartir algunas reflexiones surgidas de dos trabajos en los que confluyeron nuestras preocupaciones. Por un lado, nos basamos en un proyecto colectivo cuyo objetivo fue indagar los diversos modos en que estudiantes de los primeros años de la Universidad Nacional de Rosario (UNR) enfrentaron la virtualización forzosa impuesta por la inédita situación de pandemia (Pierella et al., 2020). Por otro lado, consideramos experiencias de formación docente propiciadas por el Sistema Institucional de Educación a Distancia (SIED-UNR).

\section{Aproximaciones metodológicas}

En relación con las experiencias estudiantiles, encaramos una indagación en la UNR, institución que cuenta con más de 85.000 estudiantes, con casi 20.000 que se inscriben anualmente en la última década (Universidad Nacional de Rosario, 2020). Implementamos un cuestionario a través de un formulario virtual destinado a estudiantes de 
primer y segundo año con preguntas cerradas y abiertas. Los ejes de indagación guardaron relación con las condiciones sociotécnicas para llevar adelante las actividades virtuales, el acceso a la nueva cultura institucional, el vínculo con docentes y pares, la organización de los propios tiempos para las tareas académicas, las valoraciones sobre las ventajas y desventajas de la modalidad de acompañamiento virtual y, por último, la dimensión afectiva, es decir, los modos en que referían a los sentimientos y emociones experimentados durante este período. La recolección de datos se produjo a mediados de 2020 y se procesaron 2500 respuestas. Para la distribución del instrumento contamos con la colaboración institucional de UNR.

\section{La recolección de datos se produjo a mediados de 2020 y se procesaron 2500 respuestas. Para la distribución del instru- mento contamos con la colaboración institucional de UNR.}

Por otra parte, la UNR organizó la formación y contención a sus docentes de diversos modos. Los espacios de formación estuvieron relacionadas con la visión institucional de la Comisión Asesora del SIED-UNR, en la que se encuentran representadas todas las Unidades Académicas que componen la UNR y que comenzara a funcionar en diciembre de 2019, es decir, solo tres meses antes del inicio del ASPO. La misma nucleó a docentes con experticia en el uso de TIC analizando y contribuyendo en la gestión de las situaciones que se iban presentando cotidianamente. Además, se gestaron espacios de formación masiva en forma de webinars y de formación continua en un espacio denominado Aulas Simplificadas con capacitaciones a demanda, foros temáticos permanentes, tutoriales específicos, clases semanales sobre temáticas relativas a la configuración en moodle y tutorías diarias (videollamadas) ante las dificultades que se iban presentando en el campus a partir de la configuración y uso de recursos y actividades. En relación a estos temas se analizan tanto cómo llegamos a la virtualización en terreno de docencia, como también las características y preocupaciones que quedaron plasmadas en instancias de capacitación durante la pandemia.

\section{¿En qué condiciones se acercaron estudiantes ingresantes y do- centes a la virtualización forzosa?}

Un primer dato se nos presentó con toda la fuerza de esos números que ponen en evidencia que algo no está bien; de 2500 estudiantes que respondieron el cuestionario, el 12\% no disponía de Internet en sus hogares, sino solo datos móviles. El $12 \%$ tenía celular para llevar 
adelante las tareas académicas; con lo complejo que es esto en términos pedagógicos (Pierella et al., 2020). Este número tiene su correlato en lo que sucede a nivel país. En Argentina, al 2020 el teléfono celular ya tenía una penetración de prácticamente 129 cada 100 habitantes, mientras que el acceso a Internet era del 65\%, con una distribución muy desigual según sector social y región (Cannellotto, 2020).

El costo de utilizar un celular sin buena conectividad para descargar tareas, ver videos, tener clases por aplicaciones de comunicación sincrónica implica tener datos. Si el acceso a datos es limitado, hay que comprarlos y no siempre se puede contar con ellos. Estas son cuestiones que, en general, se saben pero que no se suelen tener tan en cuenta cuando planificamos nuestras clases y marcan la diferencia entre la educación a distancia y la educación remota de emergencia (Hodges, et al en Dussel, 2020), que es lo que hicimos.

En este sentido, se tomaron algunas medidas como la liberación del consumo de datos para el acceso a las plataformas educativas por parte de las empresas de telefonía móvil, el decreto presidencial que establece a las prestaciones de internet y TV paga como servicios públicos (Poder Ejecutivo Nacional, 2020) y las becas brindadas por la mayoría de las universidades nacionales. Si bien todo ello ha representado un avance en materia de atender la gran brecha digital que la pandemia visibilizó; no obstante, queda mucho camino por andar para que el derecho a la conectividad se efectivice como una dimensión central de la educación superior (Pierella, 2021).

En relación con lo anterior, una de las recomendaciones de las universidades a las distintas cátedras ha sido la de utilizar plataformas y herramientas alojadas en los dominios .edu.ar; sin embargo, las instituciones educativas en general no suelen contar con programas propios para la realización de reuniones virtuales, por lo cual se ven en la necesidad de usar plataformas de empresas privadas. A su vez, se suelen "colgar" videos de clases grabadas y otros materiales didácticos que se socializan a través de las plataformas institucionales (.edu.ar) a través enlaces pero que realmente se encuentran alojados en sitios virtuales de almacenamiento comerciales. Estas situaciones, por un lado, excluyen a quienes no cuentan con acceso y conexión a internet de calidad y, por otro, someten a la universidad pública a la lógica del mercado a través de la posible comercialización de datos y publicidad.

Por su parte, el cuerpo docente se encontró con la virtualización forzosa disponiendo de escasa formación previa para el uso de TIC (Borgobello, Mandolesi et al., 2019). Incluso había cierta predominancia de lo que podría leerse como un rechazo al uso de tecnología en educación, aunque con diferencias notables entre unidades académicas. Los tiempos de ASPO exacerbaron algo que de por sí se venía dando en la relación tecnología y docencia en el nivel superior. En experiencias de formación docente para la incorporación de aulas virtuales como 
complemento del aula tradicional de clases previas a la pandemia (Borgobello, Majul et al., 2019), se vislumbraba una lectura del "tiempo como favorecedor en procesos de aprendizaje y como apremiante en términos de agenda y de dificultad del trabajo con colegas" (pp.81-82). A la vez, se destacaba la necesidad de encontrar espacios de formación y"encuentro en un contexto en el que, salvo experiencias aisladas con amplia trayectoria, el uso de aulas virtuales [estaba] basado en la voluntad individual y [era] escasamente favorecedor" (p.82).

Tal como sucediera antes del ASPO, los espacios de formación docente promovieron la solidaridad, favorecieron la motivación en el trabajo cotidiano y la discusión colectiva sobre los modos de enseñar (Borgobello, 2018), dando lugar a ese saber hacer docente que implica la construcción de un oficio que excede al conocimiento disciplinar. La docencia en tiempos de virtualización diversificó ciertos saberes "prácticos" horizontalizando y desdibujando las figuras de quienes "poseían" la experticia, no solo entre docentes, sino también entre docentes y estudiantes.

\section{La docencia en tiempos de virtualización diversificó ciertos saberes "prácticos" horizontalizando y desdibujando las figu- ras de quienes "poseían" la experticia, no solo entre docentes, sino también entre docentes y estudiantes.}

Los debates en el seno de las reuniones de SIED mostraron la diversidad que caracteriza a la UNR, con Facultades con amplia experiencia previa en EaD (Educación a Distancia) y otras con escasísima apropiación del uso del campus virtual institucional. Este panorama no difería de otras situaciones latinoamericanas. En un análisis de la educación en tiempos de pandemia a partir de narraciones de docentes de ocho países, se plasmaron preocupaciones sobre las dificultades en la organización de los tiempos; problemas de conectividad y acceso a dispositivos para el trabajo pedagógico; el propio cansancio docente a partir de la necesidad de producción de materiales específicos; insuficiente preparación tanto de estudiantes como de docentes para afrontar la enseñanza desde estas modalidades; entre otras (Proença y Borgobello, 2020).

A pesar de la cantidad y diversidad de perfiles docentes que conforman una institución con las características de la UNR, el espacio de Aulas Simplificadas mostró la necesidad de encuentros, de formación y de discusión sobre modelos y criterios en la enseñanza que superaban ampliamente la crisis generada por la forzosa virtualización. Estos espacios estuvieron caracterizados por la horizontalidad en los debates, la generación de respuestas entre pares docentes ante las múltiples y novedosas situaciones en las que se vio envuelto el trabajo pedagógico. 
Cabe destacar que el predominio de consultas docentes en la primera parte del año académico se había focalizado en aspectos vinculados a la configuración general de las aulas, de grupos, de actividades y recursos básicos. En la segunda parte del periodo académico 2020, luego del receso invernal, las consultas se enfocaron en actividades específicas destinadas a realizar evaluaciones, mostrando un importante cambio entre ambos momentos (Borgobello y Espinosa, 2020).

En paralelo a la formación de UNR, cada Facultad generó sus propios espacios de contención y formación docente. Se hicieron talleres, se multiplicaron los tutoriales con sugerencias de configuración, se crearon correos electrónicos y aulas virtuales destinadas a centralizar el asesoramiento, compartir entre colegas consultas y experiencias sobre diversas estrategias tecnopedagógicas implementadas en las diferentes cátedras (Borgobello y Espinosa, 2020).

A partir del trabajo con docentes, como se ilustra en la Figura 1, se puede afirmar que durante el 2020 la docencia se vivenció como una especie de paréntesis. Se perdieron los encuadres propios de las modalidades conocidas y sus combinaciones diversas en tiempos y espacios. Hasta entonces parecía tenerse seguridad sobre el rol docente, sus acciones posibles y las regulaciones vigentes (Borgobello y Espinosa, 2021). La crisis acarreada por la virtualización forzosa y lo que se denominó irónicamente coronateaching puso en cuestionamiento esa seguridad. Ya no sentíamos que hablábamos de lo mismo cuando decíamos aula, actividades, examen, presencia, espacio, tiempo, cara-a-cara o acompañamiento.

\section{La crisis acarreada por la virtualización forzosa y lo que se denominó irónicamente coronateaching puso en cuestiona- miento esa seguridad. Ya no sentíamos que hablábamos de lo mismo cuando decíamos aula, actividades, examen, presencia, espacio, tiempo, cara-a-cara o acompañamiento.}

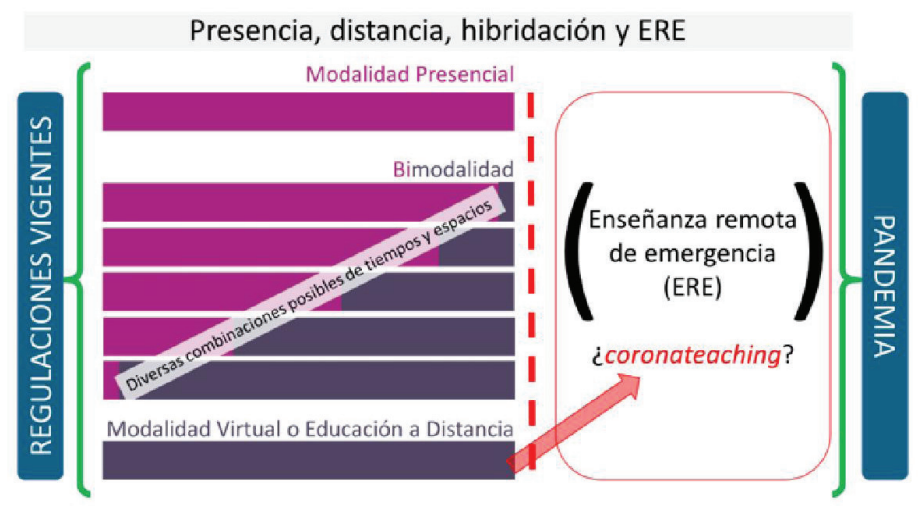

Figura 1. La enseñanza en ERE vivenciada como paréntesis (Borgobello y Espinosa, 
La UNR presentaba al momento de la irrupción del ASPO una clara tracción hacia modelos tradicionales expositivos que perdieron el encuadre en el que nos entendíamos y actuábamos: encuentros en aulas físicas, habitualmente masivos y claramente direccionados (docente-contenido- estudiantes) como se grafica en las Figuras 2 y 3.

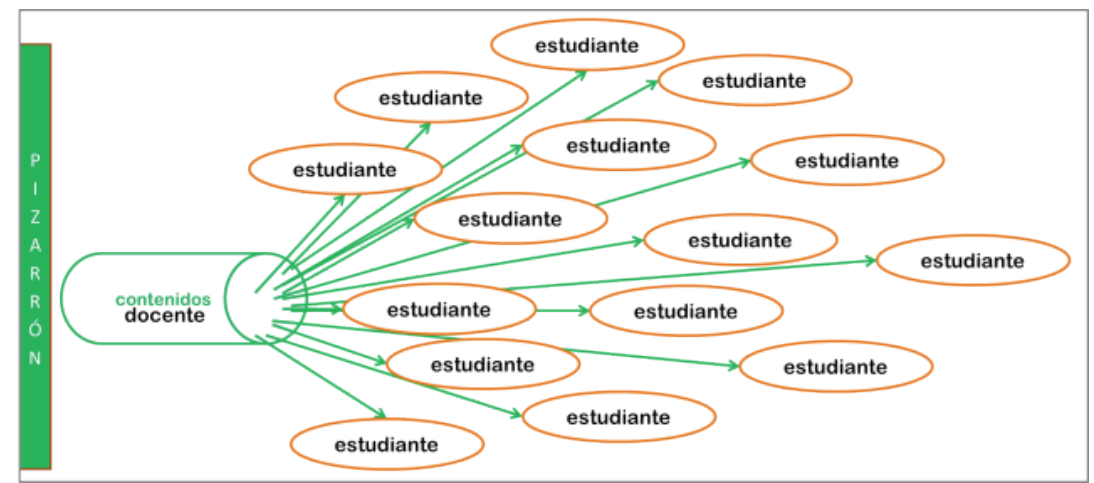

Figura 2. Encuadre tradicional

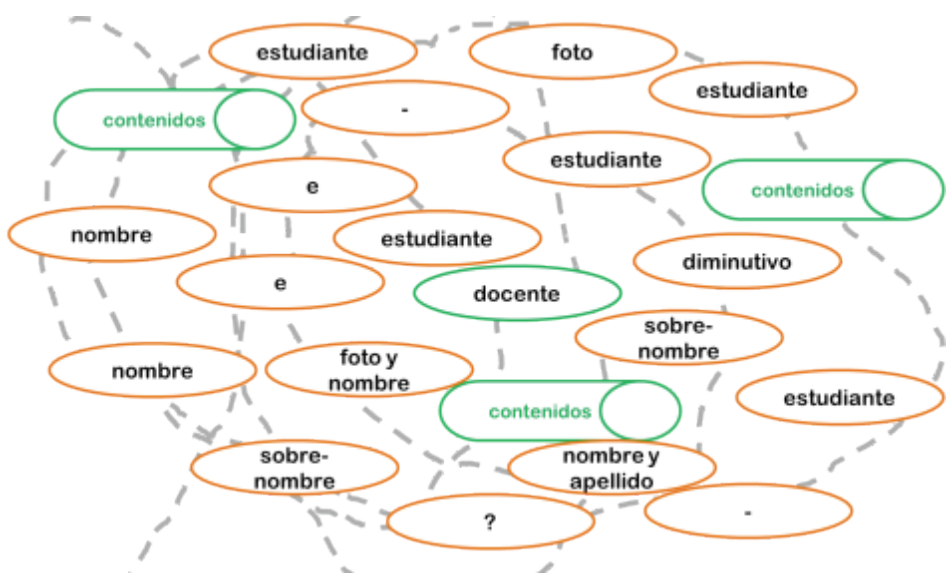

Figura 3. Se rompieron todos los encuadres conocidos (SIC estudiante, en Borgobello y Espinosa, 2021)

\section{¿Cómo fueron las experiencias educativas virtualizadas en tiempos de pandemia?}

En estudiantes de los primeros años encontramos una profunda sensación de ajenidad en relación con el conocimiento, resultando recurrentes expresiones del estilo no entiendo nada, me cuesta comprender, siento que no estoy aprendiendo y demás alusiones a esa suerte de extrañamiento que suele caracterizar a las experiencias de llegada a un universo simbólico, discursivo y disciplinar novedoso. En este caso, esto se habría encontrado agravado por una prolongación de las expectativas respecto de lo que realmente "es" la universidad. Los imaginarios acerca de dicha institución aparecen teñidos de relatos ajenos a la propia experiencia. Así, enunciaciones del estilo me contaron que, me dijeron que, 
adquirieron fuerza en la configuración de las representaciones sobre la vida universitaria, poniendo de manifiesto cierta añoranza de un espacio que aún no se conoce, o que se conoce bajo modalidades totalmente diferentes de las imaginadas (Pierella, 2021).

Como en todo habitus, en la construcción de las disposiciones subjetivas propias del oficio de estudiante, las rutinas resultan importantes. En este caso, se evidenció una gran dificultad en torno del establecimiento de ese tipo de prácticas. En este sentido, el límite necesario entre el tiempo de estudio y el tiempo de descanso parecía no poder terminar de demarcarse. La virtualidad se presentaba como una especie de presente continuo, con pocas posibilidades para establecer fronteras entre temporalidades y espacios diferentes. Esta cuestión implicó una desorganización profunda de las subjetividades y tuvo su correlato en algunas de las respuestas vinculadas a la dimensión socio-afectiva, como puede apreciarse en estos ejemplos: "Con estrés, con el sentimiento de no llegar a poder hacer todo, con el sentimiento de no entender y que se vaya acumulando textos no entendidos; "Deprimida, desganada por no poder ponerme al día"; "Pareciera que nunca hay un corte. La virtualidad está, ahí, las 24 hs. del día, toda la semana. No hay una escisión entre el aula/el hogar de cada uno de nosotros, se siente a veces un tanto invasiva".

A pesar de que predominaron las visiones negativas sobre la virtualidad, se señalaron algunos aspectos positivos que podrían sostenerse en etapas de pospandemia. Desde el punto de vista del acceso al conocimiento, se hizo alusión a la ventaja que tienen las aulas virtuales en relación con la disponibilidad de los materiales construidos y/o compartidos por docentes. La inmensa productividad pedagógica realizada durante el primer año de pandemia puso de manifiesto que no alcanzaba con saber sobre algo para saber enseñarlo. Se puso en evidencia, asimismo, la necesidad de alfabetización digital y formación pedagógica sobre todo para la docencia de los primeros años y el hecho de que el conocimiento no está dado solo por estar disponible en la red. Pero sobre todo, se visibilizó cabalmente que el conocimiento no es algo que existe por sí mismo, sino que requiere el trabajo de mediatización realizado por docentes que ofician de guías, referentes, seleccionadores, facilitadores de las trayectorias universitarias (Canellotto, 2020; Pierella, 2021).

\section{La inmensa productividad pedagógica realizada durante el primer año de pandemia puso de manifiesto que no alcanzaba con saber sobre algo para saber enseñarlo. Se puso en eviden- cia, asimismo, la necesidad de alfabetización digital y forma- ción pedagógica sobre todo para la docencia de los primeros años y el hecho de que el conocimiento no está dado solo por estar disponible en la red.}


Tomando distancia, no solo se visualizó la importancia de la enseñanza, sino que también se pudo asumir la crisis como posibilidad de innovación además de como riesgo (Proença y Borgobello, 2020). Pudo vislumbrase como una "oportunidad incalculable, única, para favorecer la independencia responsable de estudiantes como indicadores al observar, orientar y valorar los métodos de enseñanza y los sistemas de evaluación" (p.13). Mostró el resquebrajamiento de la enseñanza tradicional evidenciando que "no alienta la autonomía como cualidad a considerar y observar detenidamente" (p.13). Desde el rol docente, se dieron desafíos como aprender a manejar plataformas virtuales en contextos caracterizados por la complejidad.

Cabe señalar que a partir del segundo año de virtualización forzosa en 2021 se evidenció un segundo giro en la demanda por la formación docente en la que se pudieron capitalizar las experiencias del 2020. En el primer año de pandemia predominó la "improvisación" ante un paréntesis en la docencia que parecía tener un final inminente y una vuelta a las clases "presenciales" cercana. A diferencia del primero, el segundo año posibilitó el trabajo previo al inicio del tiempo de cursado, dando lugar al análisis de las experiencias como cátedras y a planificaciones pensadas "a largo plazo". Es posible hipotetizar que la cantidad de reuniones de cátedra, generación de contenidos, análisis del qué y cómo enseñamos dado en 2020 no pueda equipararse a ningún otro período histórico de la educación formal.

En el primer año de pandemia predominó la "improvisación" ante un paréntesis en la docencia que parecía tener un final inminente y una vuelta a las clases "presenciales" cercana. A diferencia del primero, el segundo año posibilitó el trabajo previo al inicio del tiempo de cursado, dando lugar al análisis de las experiencias como cátedras y a planificaciones pensadas "a largo plazo".

\section{Estudiantes y docentes transitando desde la crisis al después: reco- nocimiento y apropiación de los espacios en tiempos de virtualidad}

En el ingreso a la universidad podemos identificar un nudo de problemas vinculados con una dimensión central de la existencia humana, la del reconocimiento. En trabajos anteriores analizamos los diversos modos en que se pone en juego dicha aspiración básica. A partir de relatos estudiantiles sobre las experiencias de inicio, afirmábamos que esta oscila entre la valoración de las figuras profesorales que registran el nombre propio como signo de dar visibilidad e identidad a alguien y la 
legitimación del anonimato como rasgo del reconocimiento a secas sin más -el reconocimiento del estudiante como categoría que implica una dimensión de igualdad En otras palabras, señalábamos cierta exaltación de figuras a las que se les reconocía rasgos de contención y cuidado en ese tiempo de incertidumbre propio de los inicios (Pierella, 2014).En paralelo, se valoraba a la universidad pública como una institución que tiende a poner en suspenso las marcas personales y las interacciones de tipo familiar para habilitar un tipo de reconocimiento vinculado a la lógica exogámica propia de lo público (Carli, 2012; Pierella, 2014).

De nuestra investigación reciente sobre el ingreso en tiempos de pandemia se desprenden varias cuestiones para continuar pensando dichas tensiones. En primer lugar, el carácter exogámico de la institución educativa se encontraría debilitado debido a la dificultad para separar los ámbitos públicos y privados. Al mismo tiempo y en continuidad con estudios anteriores se evidenció la importancia del acompañamiento cuando este se producía, ya que se señaló como escaso. Vale aclarar que, como se ilustra en el Gráfico 1, a pesar de ser simétrico, solo tres de cada diez estudiantes marcaron alternativas positivas en cuanto al acompañamiento recibido, predominando la opción neutra en las respuestas.

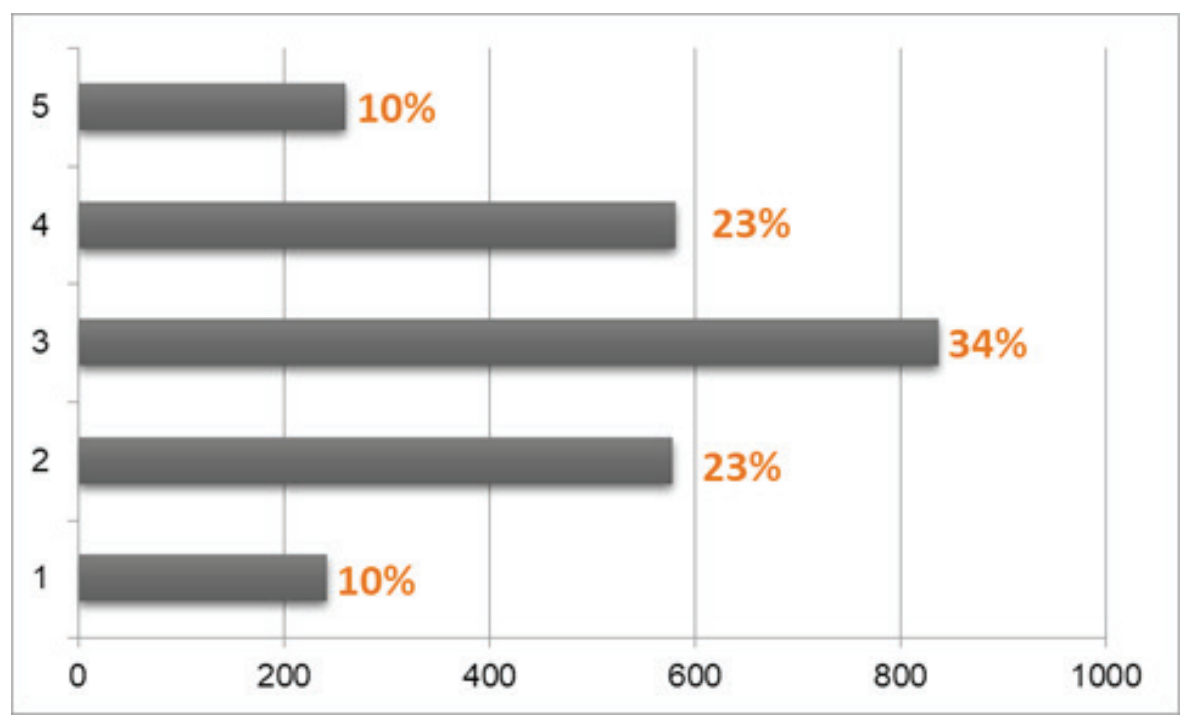

Gráfico 1. En una escala de 1 a 5, durante la cuarentena ¿cuánto te sentiste acompañado/a por tus docentes $\mathrm{u}$ otras personas de la facultad siendo $1=$ nada y $5=$ mucho $\mathrm{n}=$ 2500 (Pierella et al., 2020)

Consideramos ilustrativo respecto a los planteos hechos hasta aquí el siguiente ejemplo que sintetiza varias de las reflexiones señaladas. El mismo narra sobre diversas relaciones que se establecieron entre docentes y estudiantes: "Realmente hay muchos docentes muy predispuestos y hasta dan clases extras de consultas o nos podemos comunicar con ellos por whatsapp, mientras que otros profesores no y resulta un poco más 
distante quizás la relación, ya que nos comunicamos por mail. También hay materias en las que se respeta el programa y otras que dan mucho o nos exigen mucho más ya que estamos en casa, totalmente innecesario porque necesitamos un momento de desconexión con la facultad".

Adicionalmente, en algunos relatos frente a tantas desventajas señaladas en relación con la virtualización forzada, pudimos encontrar cierta identificación de las plataformas como espacios de mayor simetría e igualdades vinculadas al reconocimiento propio de lo público. Los sistemas de comunicación sincrónicos utilizados permitieron que las personas sean nombradas como querían ser nombradas y generando simetría: la imagen y el nombre de cada quien ocupa el mismo espacio se trate de docentes o estudiantes, "bajando al rol docente de su tarima" y sin estudiantes de primera ni última fila. Este fenómeno de descentración del rol docente se acompañó de un "golpe al ego" dada la pérdida de los encuadres propios de la docencia universitaria y en condiciones que habitualmente, como se dijo, no favorecían el trabajo pedagógico.

Líneas futuras tendrán que investigar los tránsitos, tensiones, dificultades, convivencias y potenciaciones posibles entre las experiencias educativas en tiempos de pandemia, en aulas tradicionales previas a esta crisis, aquellas que en esos tiempos se presentaban como porosas en sus muros siendo atravesadas por conexiones y dispositivos, o que se encontraban en procesos de hibridación entre presencias físicas y virtuales (e.g. Collebechi y Gobato, 2017; Dussel et al., 2020; Lion y Maggio, 2019; Villar, 2016) y los escenarios que se darán en la pospandemia.

\section{Los sistemas de comunicación sincrónicos utilizados permi- tieron que las personas sean nombradas como querían ser nombradas y generando simetría: la imagen y el nombre de cada quien ocupa el mismo espacio se trate de docentes o estudiantes, "bajando al rol docente de su tarima" y sin estu- diantes de primera ni última fila.}




\section{BIBLIOGRAFÍA}

Borgobello, A. (2018). "Realidades mentales y mundos posibles en encuentros pedagógicos presenciales y digitales". En J. Faccendini, P. Martino, M. Sironi, \& M. Terrádez (Eds.). “Caleidoscopio. Prácticas y clínicas Psi” (pp. 211-224). UNR Editora. https://rephip.unr.edu.ar/handle/2133/13830

Borgobello, A., \& Espinosa, A. (2020). “From research to intervention in educational context during pandemic times". SIP Bulletin, 107, 25-32. https://sipsych. org/publications/sip-bulletin/

Borgobello, A., \& Espinosa, A. (2021). "Enseñanza remota de emergencia: crisis, procesos y cambios en la educación superior". En S. Garo \& F. Costa (Eds.), Notas de pandemia. Reflexiones, lecturas y experiencias escritas en tiempos de aislamiento social y virtualidad (pp. 29-38). UNR Editora. https://rephip.unr.edu. ar/handle/2133/20523

Borgobello, A., Majul, S., \& De Seta, D. (2019). "Análisis cualitativo de una propuesta pedagógica para la incorporación de entornos virtuales a la enseñanza universitaria en Argentina a partir de tres ejes: experiencia, rol docente y tiempo". Hamut'ay, 6(3), 81-97. http://dx.doi.org/10.21503/hamu.v6i3.1839

Borgobello, A., Mandolesi, M., Espinosa, A., \& Sartori, M. (2019). “Uso de TIC en prácticas pedagógicas de docentes de la Facultad de Psicología de una universidad pública argentina". Revista de Psicología, 37(1), 279-317. https://doi. org/10.18800/psico.201901.010

Cannellotto, A. (2020). "Universidades viralizadas: la formación en y post pandemia". En I. Dussel, P. Ferrante y D. Pulfer, D. (comp.) Pensar la educación en tiempos de pandemia. Entre la emergencia, el compromiso y la espera (pp. 213-228). UNIPE. https://unipe.edu.ar/institucional/repositorioprensa/ item/649-pensar-la-educacion-en-tiempos-de-pandemia

Carli, S. (2012). "El estudiante universitario. Hacia una historia del presente de la educación pública". Siglo Veintiuno.

Collebechi, M. E., \& Gobato, F. (2017) (Comp.). “Formar en el horizonte digital. UVQ". Disponible en: http://libros.uvq.edu.ar

Dussel, I. (2020). "La formación docente y los desafíos de la pandemia". Revista CientíficaEFI-DGES, 6 (10) 13-25. http://ppct.caicyt.gov.ar/index.php/efi/article/view/18585

Dussel, I., Ferrante, P., \& Pulfer, D. (2020). “La educación del pasado mañana. Notas sobre la marcha". Análisis Carolina, 41(1), 1-13. https://doi.org/10.33960/ AC_41.2020 
Lion, C., \& Maggio, M. (2019). "Desafíos para la enseñanza universitaria en los escenarios digitales contemporáneos". Aportes desde la investigación. Cuadernos de Investigación Educativa, 10(1), 13-25. https://doi.org/10.18861/ cied.2019.10.1.2878

Pierella, M.P. (2014). “La autoridad en la universidad. Vínculos entre estudiantes, docentes y saberes". Paidós.

Pierella, M.P. (22 de febrero-5 de marzo de 2021). "El ingreso a la universidad en tiempos de pandemia. Experiencias estudiantiles en torno a la virtualidad". Exposición en Congreso Internacional Ingresos e Ingresantes a la Universidad, Universidad Nacional de Río Negro.

Pierella, M.P., Borgobello, A., Prados, M.L., \& Brun, L.(2020). "Ingresar a la universidad pública argentina en tiempos de virtualización forzosa". Manuscrito Enviado.

Poder Ejecutivo Nacional (2020). Decreto de Necesidad y Urgencia 690/2020 https://www.boletinoficial.gob.ar/detalleAviso/primera/233932/20200822

Proença, M., \& Borgobello, A. (2020). "Contribuições do Grupo de Trabalho Psicologia e Educação da SIP em tempos de Pandemia COVID-19". SIP Bulletin, 107, 9-14. https://sipsych.org/publications/sip-bulletin/

Universidad Nacional de Rosario (2020). Boletín estadístico $N^{\circ} 71$. Año académico 2019. https://www.unr.edu.ar/noticia/1387/boletines-estadisticos

Villar, A. (2016) (Comp.). “Bimodalidad". UVQ http://libros.uvq.edu.ar/ 